\title{
Correction
}

\section{Correction: Leão et al., Chronic Nicotine Activates Stress/Reward-Related Brain Regions and Facilitates the Transition to Compulsive Alcohol Drinking}

In the article "Chronic Nicotine Activates Stress/Reward-Related Brain Regions and Facilitates the Transition to Compulsive Alcohol Drinking” by Rodrigo M. Leão, Fábio C. Cruz, Leandro F. Vendruscolo, Giordano de Guglielmo, Marian L. Logrip, Cleopatra S. Planeta, Bruce T. Hope, George F. Koob, and Olivier George, which appeared on pages 6241-6253 of the April 15, 2015 issue, the authors regret an error in Figure 12C, where the image accidentally included 2 insets of neurons layered on top of the original image. The data and the quantification were rechecked and the mistake was found only in Figure $12 C$. The correct Figure $12 C$ is shown below. In addition, the legend should read "Green-labeled nuclei indicate Fos expression, while red-labeled cells indicate CaMKII, TH, and GAD67 labeling in panels $A, B$, and $C$, respectively" instead of "Red-labeled nuclei indicate expression of the general neuronal nuclei marker NeuN. B, Green-labeled nuclei indicate Fos expression. Merged images indicate nuclei in green”. These errors do not affect any of the conclusions or interpretations in the paper. The corrected figure and corresponding legend are provided below.
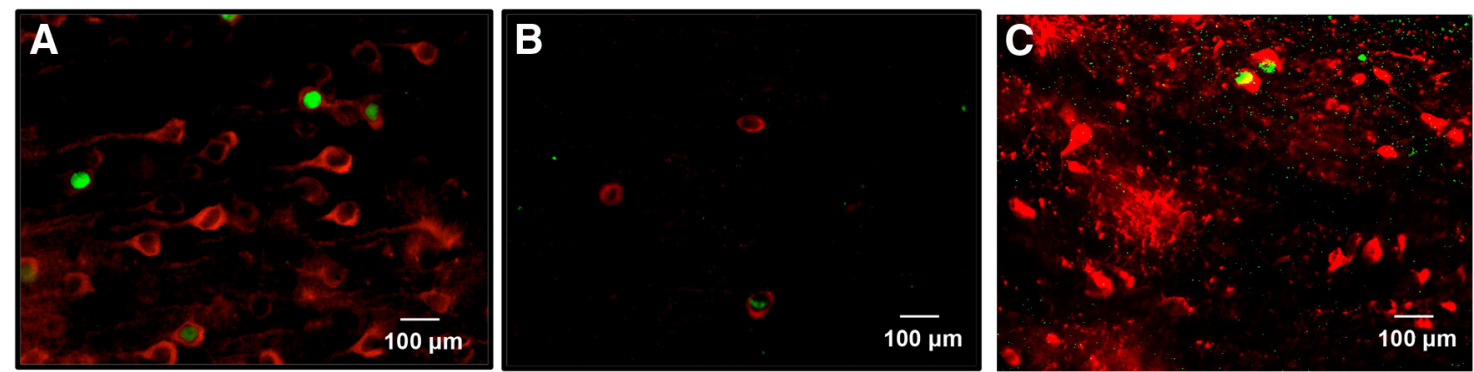

Figure 12. Representative image of double-labeled immunohistochemistry detecting Fos and CaMKII ( $\boldsymbol{A})$, Fos and TH (B), and Fos and GAD67 ( $\boldsymbol{C}$ ) associated with nicotine-induced acceleration of the escalation of alcohol self-administration. Green-labeled nuclei indicate Fos expression, while red-labeled cells indicate CaMKII, TH, and GAD67 labeling in panels $\boldsymbol{A}, \boldsymbol{B}$, and $\boldsymbol{C}$, respectively. Scale bar, $100 \mu \mathrm{m} . n=4$ per group.

DOI:10.1523/JNEUROSCI.2385-15.2015 